\title{
Asymmetric Synthesis of (+)-Hinokinin, (+)-Dihydrocubebin and Cubebin Dimethyl Ether, a New Lignan from Phyllanthus niruri
}

\author{
Dieter Enders and Mile Milovanović \\ Institut für Organische Chemie, RWTH Aachen University, Landoltweg 1, 52074 Aachen, Germany \\ Reprint requests to Prof. Dr. Dieter Enders. Fax: +49 241809 2127. E-mail: enders@ rwth-aachen.de
}

Z. Naturforsch. 2007, 62b, 117 -120; received August 23, 2006

\begin{abstract}
The asymmetric synthesis of the new lignan cubebin dimethyl ether was accomplished in eight steps with an overall yield of $40 \%$. In addition, the known lignans (+)-hinokinin and $(+)$-dihydrocubebin were synthesized by this route. Our approach involves the highly diastereoselective and enantioselective ( $d e \geq 98 \%$, ee $\geq 98 \%$ ) construction of a trans-substituted 2,3dibenzylbutyrolactone through an asymmetric Michael addition of an enantiopure lithiated aminonitrile to $5 H$-furan-2-one.
\end{abstract}

Key words: Lignans, Nucleophilic Acylation, $\alpha$-Aminonitrile, Michael Addition, Asymmetric Synthesis

\section{Introduction}

Cubebin dimethyl ether (1) is a new lignan which was recently isolated from cell suspension cultures of Phyllanthus niruri (euphorbiaceae) [1]. This plant is well-known in folk medicine as a remedy against different diseases like jaundice, asthma and bronchial infections [2]. Other lignans which were isolated from this plant also exhibited biological activities. Phyllanthin and hypophyllanthin show a moderately inhibitive effect on Grampositive and Gram-negative bacteria and anti-oxidant activities [3]. (-)-trans-2-(3,4-Dimethoxybenzyl)-3$\left(3^{\prime}, 4^{\prime}\right.$-methylenedioxybenzyl)butyrolactone, also isolated from Bursera schlechtendalii [4], showed antitumor activities and nirtetralin and niranthin are known for their anti-human hepatitis B virus activity [5].

The structure of cubebin dimethyl ether (1) was assigned on the basis of mass spectrometric combined with ${ }^{1} \mathrm{H}$ and ${ }^{13} \mathrm{C}$ NMR data and supported by a H,HCOSY, HMQC, and HMBC NMR-spectroscopic analysis. The researchers who isolated this lignan assume the $\left(8 S, 8^{\prime} S\right)$ trans-configuration due to the biogenesis of cubebin dimethyl ether [1]. Up to now exists only one report on the synthesis of $\left(8 R, 8^{\prime} R\right)$-cubebin dimethyl ether, starting from (-)-dihydrocubebin lignan and converting it in $\left(8 R, 8^{\prime} R\right)$-cubebin dimethyl ether [6].

We now wish to report the first asymmetric synthesis of cubebin dimethyl ether (1) employing our asymmetric nucleophilic acylation methodology based on<smiles>COCC(COC)[C@H](COC)Cc1ccc2c(c1)OCO2</smiles>

Cubebin dimethyl ether (1)

lithiated $\alpha$-aminonitriles [7a-h]. We first synthesized the enantiopure trans-configurated 2,3-disubstituted $\gamma$ butyrolactone $6[7 \mathrm{i}-\mathrm{j}]$, which can be easily transformed to (+)-hinokinin (8), (+)-dihydrocubebin (9) and $\left(8 S, 8^{\prime} S\right)$-cubebin dimethyl ether (1) in diastereoand enantiomerically pure form. Needless to say that employing the enantiomer of the auxiliary amine $\mathbf{2}$ should give rise to the $\left(8 R, 8^{\prime} R\right)$ enantiomer of $\mathbf{1}$.

\section{Results and Discussion}

As depicted in Scheme 1 , the $\alpha$-aminonitrile $(S, S, R / S)$-3 was obtained from the enantiomerically pure secondary amine $(S, S)$-2, piperonal and potassium cyanide in HOAc/methanol [7k]. The aminonitrile was isolated in $81 \%$ yield as a mixture of $\alpha$-epimers. Deprotonation of 3 with LDA in THF and reaction with $5 H$-furan-2-one at $-78^{\circ} \mathrm{C}$ afforded the Michael adduct $(S, S, R, R)-4$ in high yield $(79 \%)$ and diastereoselectivity $(d e \geq 88 \%$, after chromatography $d e \geq 98 \%)$.

The second stereogenic centre of the natural compound was introduced by subsequent metalation of 


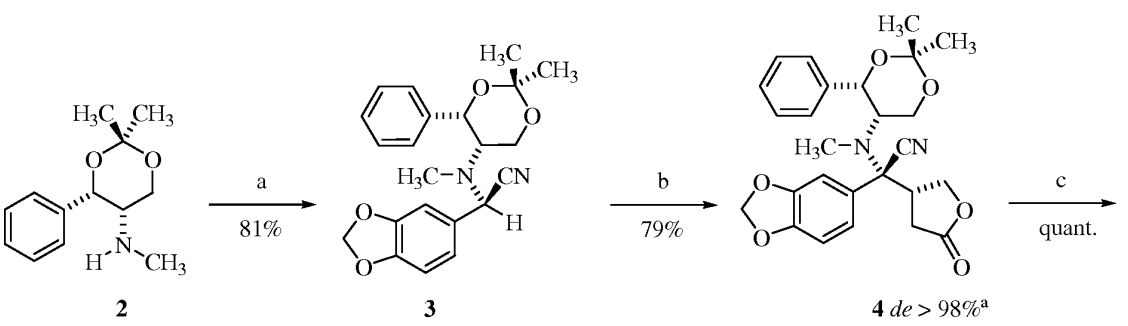

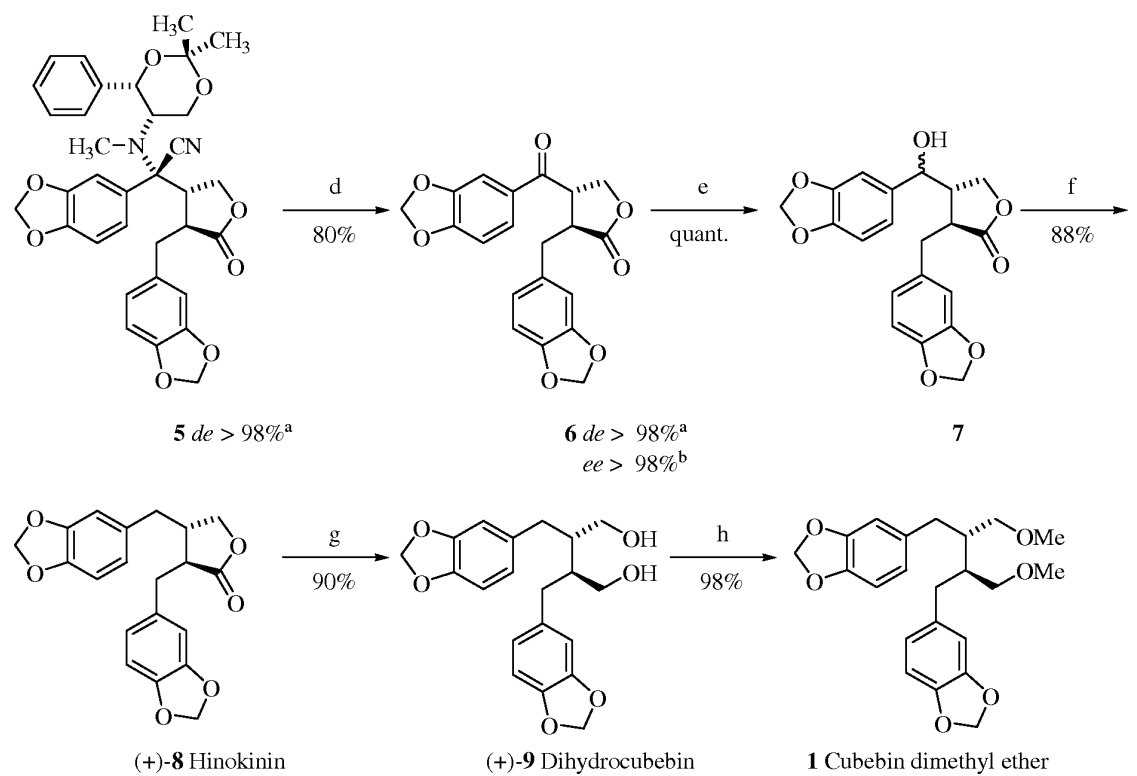

the Michael adduct 4 with 1.2 equivalents of $t$-BuLi in THF at $-100{ }^{\circ} \mathrm{C}$ and trapping of the enolate with 3,4-methylenedioxybenzyl bromide at this temperature. The resulting aminonitrile $(S, S, R, R, S)-5$ was obtained in quantitative yield and high diastereomeric purity $(d e \geq 98 \%$ ). The silver nitrate mediated cleavage of the auxiliary provided the enantiopure trans2,3-disubstituted $\gamma$-butyrolactone $(S, S)$-6 with excellent asymmetric induction ( $d e \geq 98 \%$, ee $\geq 98 \%$ ) $[7 \mathrm{~g}-\mathrm{i}]$. First the reduction of the ketone $\mathbf{6}$ with sodium borohydride in methanol quantitatively gave the corresponding alcohol 7 as an epimeric mixture. The second step, catalytic hydrogenolysis with $\mathrm{Pd} / \mathrm{C}$ at 4 atm, furnished (+)-hinokinin (8) in very good yield $(88 \%)$ and excellent stereoisomeric purity $d e \geq 98 \%$, $[\alpha]_{\mathrm{D}}^{22}=+33.0\left(c=1.6, \mathrm{CHCl}_{3}\right)$ (lit. [9]: $[\alpha]_{\mathrm{D}}^{24}=+32.8$ $\left.\left(c=1.88, \mathrm{CHCl}_{3}\right)\right)$. The subsequent reduction step with lithium aluminium hydride opened the butyrolactone ring and afforded the known lignan (+)-dihydrocubebin (9) in excellent yield $(90 \%)$ with an optical rotation of $[\alpha]_{\mathrm{D}}^{22}=+34.0\left(c=0.1, \mathrm{CHCl}_{3}\right)$ in accordance
Scheme 1. Reagents and conditions: (a) piperonal, $\mathrm{MeOH}$, $\mathrm{CH}_{3} \mathrm{CO}_{2} \mathrm{H}, \mathrm{KCN}$; (b) 1. LDA, THF, $-78{ }^{\circ} \mathrm{C}, 90 \mathrm{~min} ; 2.5 H-$ furan-2-one, $-78{ }^{\circ} \mathrm{C}$; (c) 1 . 1.2 eq. $t$-BuLi, THF, $-78{ }^{\circ} \mathrm{C}$, 90 min; 2. $\mathrm{ArCH}_{2} \mathrm{Br}, \quad-90$ to $0{ }^{\circ} \mathrm{C}$; (d) $\mathrm{AgNO}_{3}, \mathrm{H}_{2} \mathrm{O}$, THF, $25{ }^{\circ} \mathrm{C}$; (e) $\mathrm{NaBH}_{4}$, $\mathrm{MeOH} / \mathrm{CH}_{2} \mathrm{Cl}_{2}$; (f) $\mathrm{H}_{2}, \mathrm{Pd} / \mathrm{C}$, $\mathrm{HClO}_{4}, \mathrm{MeOH}, 4$ atm, rt; (g) $\mathrm{LiAlH}_{4}$, THF; (h) $\mathrm{NaH}$ (2.2 eq.), THF, MeI (8 eq.), 3 h.

a After chromatography, determined by ${ }^{1} \mathrm{H}$ and ${ }^{13} \mathrm{C}$ NMR. ${ }^{\mathrm{b}}$ Determined by ${ }^{1} \mathrm{H}$ NMR-shift reagent (Pirkle alcohol) [8].

with the literature (lit. [10]: $[\alpha]_{\mathrm{D}}^{20}=-32.4(c=3.3$, $\left.\mathrm{CHCl}_{3}\right)$ ). Finally, deprotonation of (+)-dihydrocubebin with $\mathrm{NaH}$ (2.2 eq.) and methylation with MeI (8 equiv.) provided the title cubebin dimethyl ether (1) in $98 \%$ yield. The spectroscopic data (NMR, IR, MS) were in accordance with the literature, but the optical rotations of $(S, S)-1$ differed $[\alpha]_{\mathrm{D}}^{22}=-3.1\left(c=0.1, \mathrm{CHCl}_{3}\right)$ from those given for $(R, R)-1$ (lit. [6]: $\left.[\alpha]_{\mathrm{D}}=-7.7^{\circ}\right)$.

\section{Conclusion}

In conclusion, we have reported the first asymmetric synthesis of the new lignan cubebin dimethyl ether isolated from Phyllanthus niruri Linn. (euphorbiaceae) based on our conjugate asymmetric nucleophilic acylation methodology.

\section{Experimental Section}

All products were characterized by comparison of their spectroscopic data with those of the listed literature. All moisture-sensitive reactions were carried out by using stan- 
dard Schlenk techniques. The chiral auxiliary $(S, S)-2$ was prepared according to the literature procedure [7b]. Optical rotation values were measured on a Perkin-Elmer P241 polarimeter; solvents used were of Merck UVASOL quality. Microanalyses were obtained with a Heraeus CHN-ORAPID or Vario EL element analyzer. Mass spectra were acquired on a Varian MAT 212 (EI, $70 \mathrm{eV}, 1 \mathrm{~mA})$ or Finnigan MAT SSQ 7000 (CI $100 \mathrm{eV}$ ) spectrometer. High resolution mass spectra were recorded on a Finnigan MAT 95 spectrometer. IR spectra were recorded on a Perkin-Elmer FT/IR 1760. ${ }^{1} \mathrm{H}$ NMR (300 and $400 \mathrm{MHz}$ ) and ${ }^{13} \mathrm{C}$ NMR (75 and $100 \mathrm{MHz}$ ) spectra were recorded on Gemini 300 or Varian Inova 400 spectrometers with $\mathrm{CDCl}_{3}$ as a solvent and TMS as an internal standard.

Compounds $3,4,5$, and $\mathbf{6}$ were prepared following the same procedure and experimental conditions as described previously $[7 \mathrm{k}, 7 \mathrm{~g}, 7 \mathrm{i}]$.

\section{(+)-Hinokinin (8)}

To a solution of ketone $\mathbf{6}(354 \mathrm{mg}, 0.96 \mathrm{mmol})$ in $\mathrm{CH}_{2} \mathrm{Cl}_{2}$ (30 mL) was added $\mathrm{NaBH}_{4}(0.12 \mathrm{~g}, 3.04 \mathrm{mmol})$ and $\mathrm{MeOH}$ $(15 \mathrm{~mL})$. After $2 \mathrm{~h}$ the reaction mixture was diluted with $\mathrm{CH}_{2} \mathrm{Cl}_{2}(30 \mathrm{~mL})$ and water $(10 \mathrm{~mL})$. The aqueous phase was extracted three times with $\mathrm{CH}_{2} \mathrm{Cl}_{2}(15 \mathrm{~mL})$. The combined organic layers were dried over $\mathrm{MgSO}_{4}$ and evaporated in vacuum. The crude product was directly used for the next step. The epimeric mixture of alcohol 7 (356 $\mathrm{mg}, 0.96 \mathrm{mmol})$ was dissolved in dry ethanol $(60 \mathrm{~mL})$ and two drops of aqueous $\mathrm{HClO}_{4}$ were added. The mixture was hydrogenated using $\mathrm{Pd} / \mathrm{C}(60 \mathrm{mg})$ as a catalyst at $4 \mathrm{~atm} \mathrm{H}_{2}$ pressure. After $48 \mathrm{~h}$, the solution was neutralized with $\mathrm{Na}_{2} \mathrm{CO}_{3}$ and the catalyst was filtered off. The solvent was evaporated in vacuum and the crude product was purified by column chromatography $\left(\mathrm{Et}_{2} \mathrm{O}:\right.$ pentane $\left.=1: 1\right)$ to give $299 \mathrm{mg}(88 \%)$ of (+)-hinokinin $(\mathbf{8})$ as a colourless syrup. - IR $\left(\mathrm{CHCl}_{3}\right)$ : $v=2903$ (s), 2362 (m), 1768 (s, C=O), 1492 (s), 1443 (s), 1247 (s), 1191 (s), 1038 (s), 928 (s), 811 (m), 756 (m), $668(\mathrm{~m}) .-{ }^{1} \mathrm{H}$ NMR $\left(400 \mathrm{MHz}, \mathrm{CDCl}_{3}\right): \delta=2.41-2.62$ (m, $\left.4 \mathrm{H}, \mathrm{OCH}_{2} \mathrm{CHCH}_{2}, \mathrm{OCH}_{2} \mathrm{CHCH}_{2}, \mathrm{OCCHCH}_{2}\right), 2.84$ $(\mathrm{dd}, J=14.0,7.1 \mathrm{~Hz}, 1 \mathrm{H}, \mathrm{OCCHCHH}), 2.98(\mathrm{dd}, J=14.0$, $4.9 \mathrm{~Hz}, 1 \mathrm{H}, \mathrm{OCCHCH} H), 3.85(\mathrm{dd}, J=9.0,7.4 \mathrm{~Hz}, 1 \mathrm{H}$, $\mathrm{OCHHCH}), 4.12(\mathrm{dd}, J=9.0,7.1 \mathrm{~Hz}, 1 \mathrm{H}, \mathrm{OCH} H \mathrm{CH}), 5.93$ $\left(\mathrm{s}, 4 \mathrm{H}, 2 \times \mathrm{OCH}_{2} \mathrm{O}\right), 6.46(\mathrm{~m}, 2 \mathrm{H}$, arom. $\mathrm{CH}), 6.60(\mathrm{dd}$, $J=7.9,1.6 \mathrm{~Hz}, 1 \mathrm{H}$, arom. $\mathrm{CH}), 6.62(\mathrm{~d}, J=1.6 \mathrm{~Hz}, 1 \mathrm{H}$, arom. $\mathrm{CH}), 6.69(\mathrm{~d}, J=8.2 \mathrm{~Hz}, 1 \mathrm{H}$, arom. $\mathrm{CH}), 6.73(\mathrm{~d}$, $J=7.7 \mathrm{~Hz}, 1 \mathrm{H}$, arom. $\mathrm{CH}) \mathrm{ppm} .-{ }^{13} \mathrm{C} \mathrm{NMR}(100 \mathrm{MHz}$, $\left.\mathrm{CDCl}_{3}\right): \delta=34.76\left(\mathrm{CH}_{2} \mathrm{CHCO}\right), 38.29\left(\mathrm{CH}_{2} \mathrm{CHCH}_{2} \mathrm{O}\right)$, $41.22\left(\mathrm{CH}_{2} \mathrm{CHCH}_{2}\right), 46.40\left(\mathrm{CH}_{2} \mathrm{CHCO}\right), 71.02\left(\mathrm{CHCH}_{2} \mathrm{O}\right)$, $100.86\left(\mathrm{OCH}_{2} \mathrm{O}\right), 108.10,108.17,108.64,109.26,121.35$, 122.03 (arom. CH), 131.13, 131.41, 146.12, 146.24, 147.63, 147.66 (arom. C), 178.14 (CO) ppm. - MS (EI, $70 \mathrm{eV}$ ): $\mathrm{m} / \mathrm{z}$ $(\%)=355(15)[\mathrm{M}+1]^{+}, 354(67) \mathrm{M}^{+}, 219(6), 217(16)$, 172 (6), 161 (8), 160 (7), 135 (33), 134 (100), 130 (7),
105 (5), 77 (15). $-\mathrm{C}_{20} \mathrm{H}_{18} \mathrm{O}_{6}$ (354.35): calcd. C 67.79, H 5.12; found C 68.05, H 5.23.

\section{(+)-Dihydrocubebin (9)}

To a suspension of $\mathrm{LiAlH}_{4}(28 \mathrm{mg}, 0.74 \mathrm{mmol})$ in dry THF $(5 \mathrm{~mL})$ at $0{ }^{\circ} \mathrm{C}$ under Ar, a solution of (+)-hinokinin $(130 \mathrm{mg}, 0.37 \mathrm{mmol})$ in dry THF $(5 \mathrm{~mL})$ was slowly added via syringe. The reaction mixture was stirred at $0{ }^{\circ} \mathrm{C}$ for $0.5 \mathrm{~h}$ and then at r.t. for further $0.5 \mathrm{~h}$. After addition of EtOAc $(20 \mathrm{~mL})$ the reaction mixture was quenched with saturated aqueous $\mathrm{NH}_{4} \mathrm{Cl}(10 \mathrm{~mL})$ and water $(5 \mathrm{~mL})$. The water phase was separated and extracted three times with EtOAc $(10 \mathrm{~mL})$. The combined organic layers were dried over $\mathrm{MgSO}_{4}$ and evaporated in vacuum. The crude product was purified by column chromatography $\left(\mathrm{Et}_{2} \mathrm{O}\right)$ to give (+)-dihydrocubebin (9) $(119 \mathrm{mg}, 90 \%)$ as colourless cubes. - M.p. $102{ }^{\circ} \mathrm{C}$ (lit. [11]: $\left.103-104^{\circ} \mathrm{C}\right) .-\mathrm{IR}(\mathrm{KBr}): v=3852$ (s), 3742 (s), 3681 (m), 3430 (m), 3337 (m), 2361 (s), 2339 (s), 1700 (s), 1650 (s), 1505 (s), 1246 (s), 1036 (s), 669 (m). $-{ }^{1} \mathrm{H}$ NMR (300 MHz, $\left.\mathrm{CDCl}_{3}\right): \delta=1.83\left(\mathrm{~m}, 2 \mathrm{H}, 2 \times \mathrm{CH}_{2} \mathrm{CHCH}_{2}\right.$ ), $2.60(\mathrm{dd}, J=13.6,5.6 \mathrm{~Hz}, 2 \mathrm{H}, 2 \times \mathrm{CCHH}), 2.73(\mathrm{dd}, J=$ $13.6,8.6 \mathrm{~Hz}, 2 \mathrm{H}, 2 \times \mathrm{CCH} H), 3.48(\mathrm{dd}, J=11.1,3.9 \mathrm{~Hz}$, $2 \mathrm{H}, 2 \times \mathrm{CHHOH}), 3.76(\mathrm{~d}, J=11.1 \mathrm{~Hz}, 2 \mathrm{H}, 2 \times \mathrm{CH} H \mathrm{OH})$, $3.83\left(\mathrm{~s}, 2 \mathrm{H}, 2 \times \mathrm{CH}_{2} \mathrm{OH}\right), 5.90\left(\mathrm{~s}, 4 \mathrm{H}, 2 \times \mathrm{OCH}_{2} \mathrm{O}\right), 6.59$ (d, $J=7.9 \mathrm{~Hz}, 2 \mathrm{H}$, arom. $\mathrm{CH}), 6.63$ (s, 2H, arom. $\mathrm{CH}$ ), $6.70(\mathrm{~d}, J=7.9 \mathrm{~Hz}, 2 \mathrm{H}$, arom. $\mathrm{CH}) \mathrm{ppm} .-{ }^{13} \mathrm{C} \mathrm{NMR}$ $\left(75 \mathrm{MHz}, \mathrm{CDCl}_{3}\right): \delta=35.89\left(\mathrm{CCH}_{2}\right), 44.26\left(\mathrm{CH}_{2} \mathrm{CHCH}_{2}\right)$, $60.02\left(\mathrm{CH}_{2} \mathrm{OH}\right), 100.77\left(\mathrm{OCH}_{2} \mathrm{O}\right), 108.09,109.33,121.86$ (arom. $\mathrm{CH}$ ), 134.36, 145.69, 147.55 (arom. $C$ ) ppm. - MS $(\mathrm{EI}, 70 \mathrm{eV}): m / z(\%)=359(7)[\mathrm{M}+1]^{+}, 358(38) \mathrm{M}^{+}$, 340 (14), 203 (22), 191 (10), 187 (14), 174 (5), 172 (8), 160 (6), 136 (34), 135 (100), 130 (5), 77 (10). - $\mathrm{C}_{20} \mathrm{H}_{22} \mathrm{O}_{6}$ (358.39): calcd. C 67.03, H 6.19; found C 67.27, H 6.12.

\section{Cubebin dimethyl ether (1)}

To a stirred solution of (+)-dihydrocubebin (9) $(60 \mathrm{mg}$, $0.20 \mathrm{mmol})$ in dry THF $(5 \mathrm{~mL})$ methyl iodide $(123 \mathrm{mg}$, $0.86 \mathrm{mmol}$ ), sodium hydride $(370 \mathrm{mg}, 9.20 \mathrm{mmol}, 60 \%$ dispersion in oil) and a second portion of methyl iodide $(68 \mathrm{mg}$, $0.48 \mathrm{mmol}$ ) were added. After $2.5 \mathrm{~h}$ at r.t., the mixture was cooled to $0{ }^{\circ} \mathrm{C}$ and methanol $(5 \mathrm{~mL})$ was added. Concentration under reduced pressure and flash chromatography of the crude compound $\left(\mathrm{Et}_{2} \mathrm{O}:\right.$ pentane $\left.=1: 3\right)$ afforded $76 \mathrm{mg}$ $(98 \%)$ of cubebin dimethyl ether (1) as a colourless oil. - IR $\left(\mathrm{CHCl}_{3}\right): v=2889(\mathrm{~s}), 2356(\mathrm{~s}), 1493$ (s), 1477 (s), 1246 (s), $1195(\mathrm{~m}), 1111$ (s), 1040 (s), 933 (m), 866 (s), $808(\mathrm{~m})$, 758 (s). $-{ }^{1} \mathrm{H} \mathrm{NMR}\left(400 \mathrm{MHz}, \mathrm{CDCl}_{3}\right): \delta=2.01(\mathrm{~m}, 2 \mathrm{H}, 2 \times$ $\left.\mathrm{CH}_{2} \mathrm{CHCH}_{2}\right), 2.56(\mathrm{dd}, J=13.7,8.2 \mathrm{~Hz}, 2 \mathrm{H}, 2 \times \mathrm{CCHH}$ ), 2.65 (dd, $J=13.7,6.0 \mathrm{~Hz}, 2 \mathrm{H}, 2 \times \mathrm{CCH} H), 3.28(\mathrm{~s}, 6 \mathrm{H}$, $\left.2 \times \mathrm{CH}_{3} \mathrm{O}\right), 3.28\left(\mathrm{dd}, J=9.9,4.9 \mathrm{~Hz}, 4 \mathrm{H}, 2 \times \mathrm{CH}_{3} \mathrm{OCH}_{2}\right)$, $5.91\left(\mathrm{~s}, 4 \mathrm{H}, 2 \times \mathrm{OCH}_{2} \mathrm{O}\right), 6.56(\mathrm{dd}, J=7.9,1.6 \mathrm{~Hz}, 4 \mathrm{H}$, arom. $\mathrm{CH}), 6.70(\mathrm{~d}, J=7.9 \mathrm{~Hz}, 2 \mathrm{H}$, arom. $\mathrm{CH}) \mathrm{ppm}$. ${ }^{13} \mathrm{C}$ NMR $\left(100 \mathrm{MHz}, \mathrm{CDCl}_{3}\right): \delta=34.81\left(\mathrm{CCH}_{2}\right), 40.89$ 
$\left(\mathrm{CH}_{2} \mathrm{CHCH}_{2}\right), 58.60\left(\mathrm{CH}_{3} \mathrm{O}\right), 72.39\left(\mathrm{CH}_{3} \mathrm{OCH}_{2}\right), 100.54$ $\left(\mathrm{OCH}_{2} \mathrm{O}\right), 107.76,109.25,121.70$ (arom. $\mathrm{CH}$ ), 134.67, 145.31, 147.22 (arom. C) ppm. - MS (EI, $70 \mathrm{eV}): \mathrm{m} / \mathrm{z}(\%)=$ $387(19)[\mathrm{M}+1]^{+}, 386(78) \mathrm{M}^{+}, 355$ (12), 354 (54), 322 (19), 219 (8), 217 (14), 206 (5), 204 (5), 188 (5), 186 (43), 185 (8), 173 (15). - HRMS: calcd. 386.172938; found 386.172940.

\section{Acknowledgements}

This work was supported by the Fonds der Chemischen Industrie. We thank BASF AG, Bayer AG and the former Boehringer Mannheim $\mathrm{GmbH}$ for the donation of chemicals.
[1] Elfahmi, S. Batterman, A. Koulman, T. Hackl, R. Bos, O. Kayser, H. J. Woerdenbag, W. J. Quax, J. Nat. Prod. 2006, 69, 55 - 58.

[2] L. R. Row, C. Srinivasulu, M. Smith, G. S. R. Subba Rao, Tetrahedron Lett. 1964, 5, 1557-1567.

[3] V. D. Nguyen, H. N. Luu, D. C. Nguyen, Tap Chi Duoc Hoc 2003, 12 - 14.

[4] P. Satyanarayana, S. Venkateswarlu, Tetrahedron 1991, 47, $8931-8940$.

[5] R.-L. Huang, Y.-L. Huang, J.-C. Ou, C.-C. Chen, F.-L. Hsu, C. Chang, Phytotherapy Res. 2003, 17, 449-453.

[6] A.S.R. Anjaneyulu, P.A. Ramaiah, L.R. Row, R. Venkateswarlu, A. Pelter, R. S. Ward, Tetrahedron 1981, 37, $3641-3652$.

[7] For a review on $\alpha$-aminonitrile chemistry, see: a) D. Enders, J.P. Shilvock, Chem. Soc. Rev. 2000, 29, $359-$ 373; b) D. Enders, H. Lotter, N. Maigrot, J. P. Mazaleyrat, Z. Welvart, Nouv. J. Chim. 1984, 8, 747-750; c) D. Enders, P. Gerdes, H. Kipphardt, Angew. Chem. 1990, 102, 226-228; Angew. Chem. Int. Ed. Engl. 1990, 29, 179-181; d) G. Raabe, E. Zobel, J. Fleischhauer, P. Gerdes, D. Mannes, E. Müller,
D. Enders, Z. Naturforsch. 1991, 46a, 275-288; e) D. Enders, D. Mannes, G. Raabe, Synlett 1992, 837 839; f) D. Enders, J. Kirchhoff, D. Mannes, G. Raabe, Synthesis 1995, 659-666; g) D. Enders, J. Kirchhoff, V. Lausberg, Liebigs Ann. 1996, 1361 - 1366; h) D. Enders, J. Kirchhoff, P. Gerdes, D. Mannes, G. Raabe, J. Runsink, G. Boche, M. Marsch, H. Ahlbrecht, H. Sommer, Eur. J. Org. Chem. 1998, 63 - 72. For previous lignan syntheses of our group, see: i) D. Enders, V. Lausberg, G. Del Signore, O. M. Berner, Synthesis 2002, 515-522; j) D. Enders, G. Del Signore, O. M. Berner, Chirality 2003, 15, 510 - 513; k) D. Enders, M. Milovanović, E. Voloshina, G. Raabe, J. Fleischhauer, Eur. J. Org. Chem. 2005, 1984-1990.

[8] W.H. Pirkle, D. L. Sikkenga, M. S. Pavlin, J. Org. Chem. 1977, 42, $384-387$.

[9] T. Morimoto, H. Nagai, K. Achiwa, Synth. Comm. 2005, 35, 857-865.

[10] J. E. Batterbee, R. S. Burden, L. Crombie, D. A. Whiting, J. Chem. Soc. (C) 1969, $2470-2477$.

[11] G. Bruchhausen, Chem. Ber. 1939, 72, 830-836. 